\title{
The use of local isolates in Western blots improves serological diagnosis of Lyme disease in Scotland
}

\author{
Correspondence \\ S. Mavin \\ microbiology@haht.scot.nhs.uk
}

Received 22 June 2006

Accepted 22 September 2006

\author{
S. Mavin, R. M. Milner, R. Evans, J. M. W. Chatterton, A. W. L. Joss \\ and D. O. Ho-Yen
}

Microbiology Department, Raigmore Hospital, Old Perth Road, Inverness IV2 3UJ, UK
Nine Scottish Borrelia burgdorferi isolates were investigated in lgG Western blot tests. Sera previously found to be positive and negative when tested by routine Western blots prepared from reference strain $B$. burgdorferi sensu stricto antigen had different outcomes with these isolates. Two isolates, E5 (Borrelia afzelii) and G4 (B. burgdorferi sensu stricto) performed well, reproducing Western blot-positive results in 90 and $95 \%$ of tests, respectively. When antigens from both isolates were incorporated into a single IgG Western blot, the results of a panel of sera were improved when compared to the routine reference strain lgG Western blot. All of the sera positive by the routine Western blot remained positive using the Scottish isolate antigen mix. Twenty-three of the 25 negative sera remained negative and two produced an equivocal result. Of the 15 samples that tested IgG Western blot equivocal with the $B$. burgdorferi sensu stricto reference strain, $11(73 \%)$ became weak or strong positive when tested with the $B$. afzeliilB. burgdorferi sensu stricto antigen mix $\left(\chi^{2}=14.35\right.$, Yates' correction, $\left.P<0.001\right)$. In seven of these, a clinical picture of Lyme disease was consistent with the new results. The use of Scottish strains of $B$. afzelii and $B$. burgdorferi sensu stricto to provide antigen for the lgG Western blot improves the diagnosis of Lyme disease for patients in Scotland.

\section{INTRODUCTION}

The isolation of Borrelia burgdorferi from ticks in the Highlands of Scotland was reported by Davidson et al., (1999). Two pathogenic species from the B. burgdorferi sensu lato complex were isolated, B. burgdorferi sensu stricto and Borrelia afzelii. The third pathogenic species that has been previously isolated from patients in Europe with Lyme disease, Borrelia garinii (Wilske 2003), was not isolated. Recommended practice for the laboratory diagnosis of Lyme disease is a two-step procedure, involving a sensitive screening enzyme immunoassay (EIA), followed by a Western blot to confirm EIA-positive or equivocal results (Wilske 2003). Locally, Western blot may also be performed on EIA-negative samples from patients with clinical symptoms consistent with early Lyme disease (Davidson et al., 1996). Some patients with clinical evidence of Lyme disease have been found to be EIA negative and Western blot positive.

The presence of only one pathogenic species, B. burgdorferi sensu stricto, in the USA has allowed the Western blot test to be well standardized (Centers for Disease Control, 1995; Hauser et al., 1997). In Europe it has been more problematic. Strain and protocol variation has meant that a consensus has not been reached (Hauser et al., 1998;

Abbreviations: EIA, enzyme immunoassay; EM, erythema migrans.
Robertson et al., 2000; Heikkila et al., 2002). Historically, the Western blot test utilized by the National Lyme Disease Testing Service Laboratory at Raigmore Hospital, Inverness, UK, has been based on antigen prepared from a reference strain of B. burgdorferi sensu stricto. However, there is concern that false-negative Western blot results may be reported, particularly from patients with acute symptoms of Lyme disease. Although false negatives may be a result of treatment, or of testing a patient too soon after infection, the strain of B. burgdorferi used in diagnostic assays has been shown to influence test sensitivity (Hauser et al., 1997; Kaiser 2000; Robertson et al., 2000). In this study, antigen from Scottish B. burgdorferi isolates was incorporated into an IgG Western blot, and the results were compared with those of the reference strain of B. burgdorferi sensu stricto currently used in the Western blot test.

\section{METHODS}

B. burgdorferi culture/antigen preparation. Nine Scottish isolates of B. burgdorferi, which had been stored in liquid nitrogen, were thawed rapidly and cultured in $7 \mathrm{ml} \mathrm{screw-capped} \mathrm{glass} \mathrm{bottles}$ and $25 \mathrm{~cm}^{2}$ flasks with Barbour-Stoenner-Kelly (BSK-H) medium, as described previously (Davidson et al., 1999). The isolates were E9, G4, G5, G10 and H1 (B. burgdorferi sensu stricto), and C7, E5, F2 and F8 (B. afzelii). Once sufficient growth was achieved, the media were centrifuged at $3000 \mathrm{~g}$ for $30 \mathrm{~min}$ and the pellets washed twice in PBS. Each pellet was resuspended in $1 \mathrm{ml}$ distilled water and the antigen stored at $-70{ }^{\circ} \mathrm{C}$ until used. 
Antigen selection. A panel of human sera (panel A) was selected to assess the suitability of antigen from each of the nine Scottish isolates for the detection of B. burgdorferi IgG antibodies in a Western blot. The sera panel included: one commercial B. burgdorferi-positive control (EIA and IgG Western blot strong positive, Zeus Scientific), nine $B$. burgdorferi EIA/IgG Western blot positive/strong positive from patients with acute symptoms of Lyme disease [tick bite, erythema migrans (EM)/rash] and six B. burgdorferi EIA/IgG Western blot negative from patients with non-specific clinical symptoms (muscle pains and demyelination). Previous IgG Western blot results were from testing with reference strain $\mathrm{Hb} 1 \mathrm{~B}$. burgdorferi sensu stricto antigen in the routine testing system.

Western blotting. Western blots were prepared from antigen from all nine Scottish isolates, as described previously (Davidson et al., 1996). SDS 7 molecular mass marker (14 200-66000 Da range, Sigma) was run on each gel, stained with Amido Black and used as a standard to determine the molecular masses of test proteins. Western blotting was performed on serum panel A as described previously (Davidson et al., 1996), using blots prepared from each of the nine Scottish antigens. Where possible, each serum was tested twice with each Western blot. Duplicate testing could not be carried out with every blot due to limited amounts of sera or antigen. The number, intensity and molecular mass of bands observed with each test were recorded, and a negative, equivocal, weak-positive, positive or strong-positive result assigned according to pre-determined criteria (Evans et al., 2005). Briefly, a negative blot has no borrelia-specific bands $(18,22,26,30,32,34,39,46,58$ and $92 \mathrm{kDa})$. The $41 \mathrm{kDa}$ band must be present for an equivocal or positive result. An equivocal result has one specific band, but fewer than four bands in total. Weak-positive/positive results require four or more bands in total. A weak positive has one strong specific band, or more than two weak specific bands, whereas positive and strong-positive samples have two and more than two specific bands, respectively, with a strong intensity.

Antigen assessment. Two isolates, one B. afzelii and one B. burgdorferi sensu stricto, were selected for further investigation and cultured as above to produce a sufficient amount of fresh antigen. Approximately $0.6 \mathrm{~g} \mathrm{l}^{-1}$ of each extract was incorporated into a Western blot. This optimal protein concentration was confirmed by testing with commercial and in-house B. burgdorferi-positive controls.

A second panel of human sera (panel B) was used to assess the two selected antigens. The sera panel consisted of: 25 B. burgdorferi EIA/IgG Western blot-negative ( 15 anonymous healthy donors, 10 neurological screening/non-specific clinical symptoms); 12 B. burgdorferi EIA/IgG Western blot-positive/strong positive (five early Lyme disease, seven late Lyme disease); and 15 B. burgdorferi IgG Western blot-equivocal samples (Table 1). Five IgG Western blots were made from the two antigens: one blot for each antigen at its optimum dilution, then three containing mixes of both antigens in different proportions $(50: 50$, $60: 40$ and $40: 60$ ). For the $50: 50 \mathrm{mix}$ the antigens were added so that each would be at its optimum working concentration, in equal amounts. The blots were tested against a small number of sera from panel B [two B. burgdorferi EIA/IgG Western blot negative (sera B1 and 2), two B. burgdorferi EIA/IgG Western blot positive/strong positive (sera B26 and 27) and five B. burgdorferi IgG Western blot equivocal (sera B38, 39, 40, 41 and 42)]. The results were compared with those previously obtained with the reference B. burgdorferi sensu stricto antigen to determine the best antigen combination. The blot made from this antigen combination was then tested with all the sera from panel B. Again, the results were compared with those obtained previously and the significance of any change in the number of sera in each result category assessed by the $\chi^{2}$ test.

\section{RESULTS AND DISCUSSION}

\section{Antigen selection}

Sera previously found to be positive and negative with the routine IgG Western blot prepared from reference strain $B$. burgdorferi sensu stricto antigen (serum panel A), produced different results with the nine Scottish B. burgdorferi isolates (Table 2). Antigen from the B. afzelii isolates (E5, F8, C7 and F2) produced fewer non-specific bands than antigen from the B. burgdorferi sensu stricto isolates (E9, G4, G5, G10 and $\mathrm{H} 1$ ), and all previously Western blot-negative sera remained negative. Only E5 and F8 antigens produced acceptable results with the sera previously found to be Western blot positive, with 18 out of 20 (90\%), and 16 out of 19 (84\%) tests, respectively, remaining weak positive or stronger. None of the Western blot-positive sera produced negative results with the E5 and F8 antigens, whereas C7 and F2 antigens produced negative results in three out of $13(23 \%)$ and one out of $13(8 \%)$ tests, respectively. Antigen from the E9 and G10 B. burgdorferi sensu stricto isolates did not produce a reaction with the Western blot-negative sera; however, they both failed to produce positive results in four out of $10(40 \%)$ and four out of $19(21 \%)$ tests on Western blot-positive sera, respectively. In contrast, the G4 and G5 antigens achieved weak-positive or stronger results in 19 out of $20(95 \%)$ and 18 out of $19(95 \%)$ positive tests, respectively. G4 and G5 antigens produced more discrepant results with the negative sera, although G4 performed better than G5, with two out of 12 (17\%), compared with five out of $11(45 \%)$ tests on Western blot-negative sera, producing equivocal results. These differences may have been due to the amount of antigen present in each blot, as this was not controlled due to the small amounts of stored antigen available. However, it is likely that the differences were a result of strain variation. The different isolates had quite different growth characteristics in culture (Davidson et al., 1999). In addition, the nine Western blot-positive samples in serum panel A were originally detected with the routine $B$. burgdorferi sensu stricto test, but the species that caused the infection had not been identified.

\section{Antigen assessment}

Two isolates, E5 (B. afzelii) and G4 (B. burgdorferi sensu stricto), were selected for further investigation based on their ability to produce the appropriate IgG Western blot-positive and -negative results. The inclusion of antigen from both $B$. afzelii and B. burgdorferi sensu stricto Scottish isolates in the same Western blot could increase the detection of Lyme disease. Kaiser (2000), from a study of false-negative serology in patients with neuroborreliosis, concluded that a negative serological result with one strain should prompt the repetition of the test with other strains from the $B$. burgdorferi sensu lato complex.

The Western blot prepared from a 50:50 mix of E5 and G4 antigen (E5/G4 50:50) performed marginally better with the Western blot-equivocal samples (selected from serum 
Table 1. Serum panel B for antigen assessment

Results: - , negative; \pm , equivocal; + , positive.

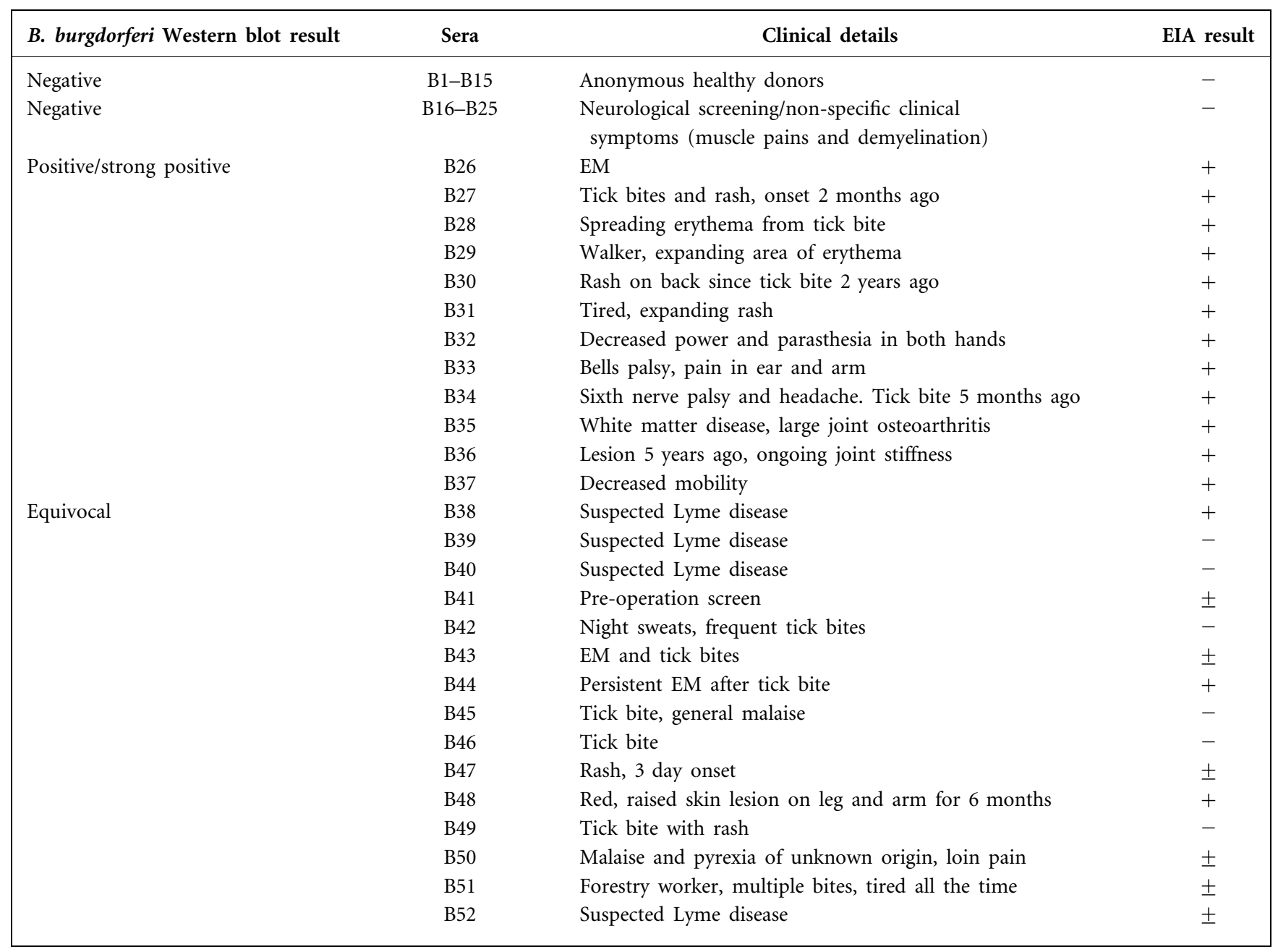

Table 2. Results of Western blot antigen selection (serum panel A)

\begin{tabular}{|c|c|c|c|c|c|c|c|c|c|c|}
\hline \multirow[t]{2}{*}{ Original Western blot result } & \multirow[t]{2}{*}{ New Western blot result } & \multicolumn{9}{|c|}{ Scottish B. burgdorferi isolates ${ }^{\star}$} \\
\hline & & \multicolumn{5}{|c|}{ B. burgdorferi sensu stricto } & \multicolumn{4}{|c|}{ B. afzelii } \\
\hline \multirow[t]{3}{*}{ Positive/strong positive (10 sera) } & Positive/strong positive & 3 & 17 & 16 & 15 & 18 & 5 & 15 & 9 & 15 \\
\hline & Weak positive & 3 & 2 & 2 & 0 & 2 & 3 & 3 & 1 & 1 \\
\hline & Total no. tests ${ }^{*}$ & 10 & 20 & 19 & $19 \dagger$ & 20 & 13 & 20 & 13 & $19 \dagger$ \\
\hline \multirow[t]{4}{*}{ Negative (6 sera) } & Weak positive & 0 & 0 & 0 & 0 & 1 & 0 & 0 & 0 & 0 \\
\hline & Equivocal & 0 & 2 & 5 & 0 & 6 & 0 & 0 & 0 & 0 \\
\hline & Negative & 6 & 10 & 6 & 12 & 5 & 7 & 12 & 7 & 12 \\
\hline & Total no. tests & 6 & 12 & 11 & 12 & 12 & 7 & 12 & 7 & 12 \\
\hline
\end{tabular}

${ }^{\star}$ Indicates number of tests (including duplicate tests), not number of sera tested.

$\dagger$ No duplicate run was carried out on some samples due to limited amount of antigen/sera. 
panel B) than the individual E5 and G4 blots and the E5/G4 (40:60) mix (Table 3), producing weak or strong-positive results in four out of the five $(80 \%)$ equivocal sera. This compared with two out of five $(40 \%)$, three out of five $(60 \%)$ and two out of four $(50 \%)$, respectively. Although all equivocal sera became weak positive with the E5/G4 (60:40) antigen mix, one negative serum produced an equivocal result. All Western blot-negative sera remained negative with the other antigen combinations. Fig. 1 illustrates an example of a change in result with the different antigens, individually and combined. Serum B38 produced an equivocal result when tested with the routine B. burgdorferi sensu stricto IgG Western blot, a weakpositive result with the individual G4 blot, and positive results with the individual E5 and E5/G4 (50:50) Western blot.

The diagnosis of Lyme disease was improved when the Western blot made from the E5/G4 (50:50) antigen mix was tested with all the serum in panel B. Compared with the routine B. burgdorferi sensu stricto IgG Western blot (Table 4), 23 of the 25 (92\%) Western blot-negative sera remained negative, and one produced an equivocal result. Similarly, 10 of the $12(83 \%)$ Western blot-positive sera remained positive, and two produced a weak-positive result. The biggest difference was seen with the sera that had previously produced equivocal results. The E5/G4 (50:50) Western blot produced weak- or strong-positive results in 11 out of the $15(73 \%)$ sera previously equivocal (Table 4$)$; this change was significant $\left(\chi^{2}=14.35\right.$, Yates' correction, $P<0.001)$.

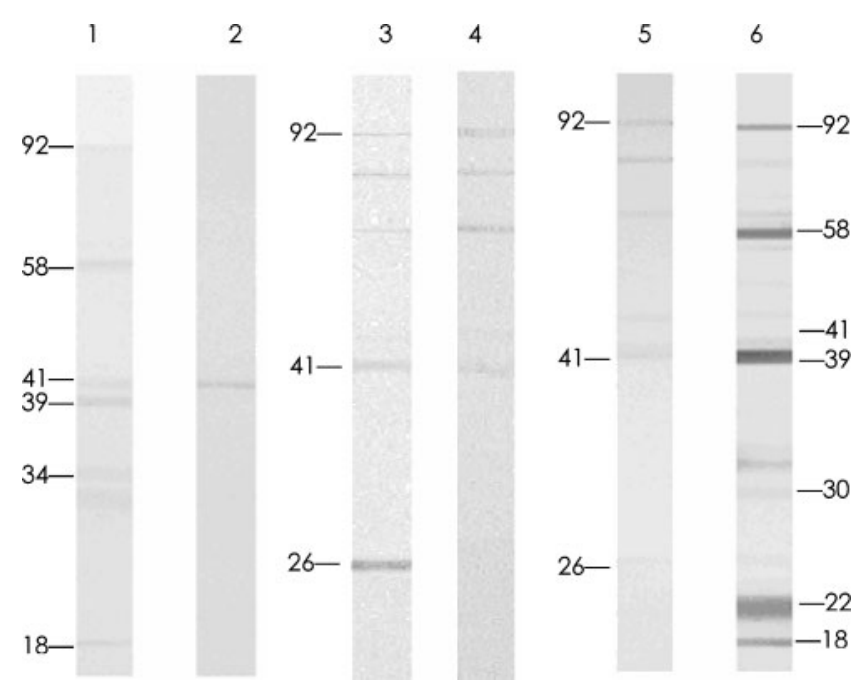

Fig. 1. Differences in Western blot result when serum B38 was tested against antigens from different Borrelia species. Numbers next to lanes show molecular mass $(\mathrm{kDa})$. Lane 1, routine $B$. burgdorferi sensu stricto antigen reacted against a commercial positive-control serum; lane 2, the same antigen reacted with serum B38 (equivocal reactivity); lane 3, Scottish antigen E5 (B. afzelii) reacted with serum B38 (positive reactivity); lane 4, Scottish antigen G4 (B. burgdorferi sensu stricto) reacted with serum $B 38$ (weak-positive reactivity); lane 5, E5/G4 (50:50) antigen mix reacted with serum B38 (positive reactivity); lane 6 , the same antigen reacted with a commercial positive-control serum.

Table 3. Antigen assessment: comparison of selected antigen combinations

\begin{tabular}{|c|c|c|c|c|c|c|}
\hline \multirow[t]{2}{*}{ Original Western blot result } & \multirow[t]{2}{*}{ New Western blot result } & \multicolumn{5}{|c|}{ Antigen combination ${ }^{\star}$} \\
\hline & & E5 & G4 & E5/G4 $(50: 50)$ & E5/G4 $(60: 40)$ & E5/G4 (40:60) \\
\hline \multirow{3}{*}{ Positive/strong positive } & Weak positive & 0 & 1 & 1 & 1 & 1 \\
\hline & Equivocal & 0 & 0 & 0 & 0 & 0 \\
\hline & Negative & 0 & 0 & 0 & 0 & 0 \\
\hline \multirow[t]{5}{*}{ Equivocal } & Positive/strong positive & 1 & 2 & 1 & 0 & \\
\hline & Weak positive & 1 & 1 & 3 & 4 & 2 \\
\hline & Equivocal & 2 & 2 & 1 & 0 & 2 \\
\hline & Negative & 1 & 0 & 0 & 0 & 0 \\
\hline & Total no. sera tested & 5 & 5 & 5 & 4 & 4 \\
\hline \multirow[t]{2}{*}{ Negative } & Positive/strong positive & 0 & 0 & 0 & 0 & 0 \\
\hline & Total no. sera tested & 2 & 2 & 2 & 2 & 2 \\
\hline
\end{tabular}

${ }^{*}$ Values show the number of sera from panel B. 
Table 4. Samples whose Western blot results changed when tested with Scottish isolate E5 (B. afzelii)/G4 (B. burgdorferi sensu stricto) $50: 50$ antigen mix

Abbreviations: - , negative; \pm , equivocal; $\mathrm{Wk}+$, weak positive; + , positive; ++ , strong positive.

\begin{tabular}{|c|c|c|c|c|c|c|}
\hline \multirow[t]{2}{*}{ Sera } & \multicolumn{6}{|c|}{ Western blot result } \\
\hline & Non-specific bands & Specific bands & Result & Non-specific bands & Specific bands & Result \\
\hline B4 & 2 & 0 & - & 2 & 1 & \pm \\
\hline B11 & 0 & 0 & - & 1 & 1 & \pm \\
\hline B38 & 2 & 1 & \pm & 5 & 2 & + \\
\hline B39 & 2 & 1 & \pm & 4 & 2 & $\mathrm{Wk}+$ \\
\hline B40 & 1 & 1 & \pm & 3 & 1 & $\mathrm{Wk}+$ \\
\hline B42 & 2 & 1 & \pm & 2 & 2 & $\mathrm{Wk}+$ \\
\hline B43 & 2 & 1 & \pm & 5 & 1 & $\mathrm{Wk}+$ \\
\hline B50 & 2 & 1 & \pm & 4 & 4 & ++ \\
\hline B51 & 3 & 2 & \pm & 7 & 3 & ++ \\
\hline
\end{tabular}

The incorporation of antigen from individual Scottish isolates of $B$. afzelii (E5) and B. burgdorferi sensu stricto (G4) into separate Western blots for the detection of $B$. burgdorferi IgG antibodies produced in both cases better discrimination of results. This improved discrimination was maintained when the antigens were combined in a single Western blot. Now, $73 \%$ of sera which were previously found to have equivocal results with routine Western blots have produced more definitive results (weak or strong positive), a significant improvement in sensitivity. The majority of patients in whom this improvement was demonstrated (seven out of 11) had a clinical picture consistent with Lyme disease, such as tick bites and/or rash (Tables 1 and 4). A Western blot which combines antigens from two of the species of B. burgdorferi known to exist in Scotland (and Europe) should reduce the risk of false-negative results. An increase in sensitivity, without compromising specificity, means that more patients with Lyme disease will be detected, and this should aid patient management.

\section{ACKNOWLEDGEMENTS}

We would like to thank all of the users of the National Lyme Disease Service for their support and input on the development of the service.

\section{REFERENCES}

Centers for Disease Control (1995). Notice to readers recommendations for test performance and interpretation from the second national conference on serologic diagnosis of Lyme disease. Morbid Mortal Wkly Rep 44, 590-591.
Davidson, M. M., Chisholm, S. M., Wiseman, A. D., Joss, A. W. L. \& Ho-Yen, D. O. (1996). Improved serodiagnosis of Lyme disease. J Clin Pathol 49, M80-M84.

Davidson, M. M., Evans, R., Ling, C. L., Wiseman, A. D., Joss, A. W. L. \& Ho-Yen, D. O. (1999). Isolation of Borrelia burgdorferi from ticks in the Highlands of Scotland. J Med Microbiol 48, 59-65.

Evans, R., Mavin, S. \& Ho-Yen, D. O. (2005). Audit of the laboratory diagnosis of Lyme disease in Scotland. J Med Microbiol 54, 1139-1141.

Hauser, U., Lehnert, G., Lobentanzer, R. \& Wilske, B. (1997). Interpretation criteria for standardized Western blots for three European species of Borrelia burgdorferi sensu lato. J Clin Microbiol 35, 1433-1444.

Hauser, U., Krahl, H., Peters, H., Fingerle, V. \& Wilske, B. (1998). Impact of strain heterogeneity on Lyme disease serology in Europe: comparison of enzyme-linked immunosorbent assays using different species of Borrelia burgdorferi sensu lato. J Clin Microbiol 36, 427-436.

Heikkila, T., Seppala, I., Saxen, H., Panelius, J., Yrjanainen, H. \& Lahdenne, P. (2002). Species-specific serodiagnosis of Lyme arthritis and neuroborreliosis due to Borrelia burgdorferi sensu stricto, $B$. afzelii and B. garinii by using decorin binding protein A. J Clin Microbiol 40, 453-460.

Kaiser, R. (2000). False-negative serology in patients with neuroborreliosis and the value of employing different borrelial strains in serological assays. J Med Microbiol 49, 911-915.

Robertson, J., Guy, E., Andrews, N., Wilske, B., Anda, P., Granstrom, M., Hauser, U., Moosmann, Y., Sambri, V. \& other authors (2000). A European multicenter study of immunoblotting in serodiagnosis of Lyme borreliosis. J Clin Microbiol 38, 2097-2102.

Wilske, B. (2003). Diagnosis of Lyme borreliosis in Europe. Vector Borne Zoonotic Dis 3, 215-227. 